these two cases, it becomes clear that any good obtained by aspiration of the fluid in the joint is an accidental good, and not one to be depended upon. Clotted blood thickly adhered to the broken surface of the upper fragment, and no amount of aspiration could have removed it. It formed another definite obstacle to immediate bony union.

The two cases were admitted under Mr. Croft's care in St. Thomas's Hospital on two following days in April, $189 \mathrm{~L}-$ viz., the 21 st and $22 \mathrm{nd}$. They were suffering from similar injuries-recent, simple, transverse fracture of the patella, the bone in each case being divided into unequal halves, the lower half being the smaller. There had not been any direct violence in either case. In both there was a swollen blood-discoloured state of the knee from considerable effusion of blood into its cavity. The separation of the fagments was about three-quarters of an inch. It appeared to $\mathrm{Mr}$. Croft and others that the pieces of bone could be pressed into very near, if not close, contact. In one case the operation was done on the day following the injury, and in the other case the operation was delayed until the fifth day on account of a mild attack of albuminuria. The patient's urine was free from albumen when the operation was performed. The operation was similar in each case, and therefore one description will serve for the two. After proper antiseptic preparation of the limb a longitudinal incision was made long enough to expose both fragments of bone, and deep enough to open up the joint. A large quantity of fluid and coagulated blood was cleared away from the torn surfaces and from the cavity of the joint. In each ase the fracture surface of the upper piece of the patella was thickly furred over with coagulum. In the fifth-day case there was a thick, firm layer of laminating clot on the fracture surface, and this could only be removed by scraping. The lower fragment was at first invisible, and anly came into view when the soft parts which had fallen in over its raw surface had been lifted out. A thick tongue-shaped piece of the aponeurosis of the external quadriceps had been torn from the surface of the upper fragment, and had become so wedged into the intercundyloid space that it quite masked the lower fragment, and it served is a complete and material obstacle to the close approxima tion of the fragments, and of course to their bony union. When, in each case, this mass of aponeurosis and fibrous tissue was held aside the cavity of the joint was cleared, and the sutures inserted. A simple suture of double stont silver wire was used on each case to suture the pieces of bone, and these were brought into close contact without any serious tension. The edges of the aponeurosis were trimmed and sutured by several silk sutures. The edges of the torn "bursa" were also trimmed, and its cavity was cleared. In each patient a counter-opening on the outer side of the knee was made to drain both bursa and joint. The wound was sutured in the ordinary way, and a small drain established on its centre. The parts were dressed antiseptically and immobilised. As regards the after progress little need be said, as the main interest about the cases is in their demonstration that the chief obstacles to bony union in instances of similar fracture of the patella is in the interposition of a more or less thick tongue of aponeurosis and fascia between the fragments. Neither man suffered any constitutional disturbance. One had a little bocal temporary uneasiness. In each case strong bony union took place in about a month's time. The younger, healthier, and pluckier man has, as might have been expected, made the more rapid recovery, and regained the use of his joint. The other man, who suffered from albuminuria at the time of the accident, and who is not at all self helpful, still needs to have passive movements of the joints forced upon him. That, however, does not affect the fact that his fractured patella has firmly reunited by bone.

\section{MEDICAL COLLEGE HOSPITAL, CALCUTTA.}

\section{ANEURYSM: SIX CASES OF LIGATCRE OF THE MAIN ARTERIES.}

(Under the care of Mr. O'CONNELL RAYE.)

PUBLISHED statistics of operations on the large arteries being mainly derived from the experience of pre-antiseptic days, are necessarily somewhat misleading to the modern surgeon. These operations being comparatively uncommon, the number falling to the lot of any individual is small, and statistics can therefore be but slowly collected. The following cases do not present any very remarkable features, but are published as a contribution to the records of more recen operations. They include all the cases of ligature of the larger vessels which occurred in Mr. Raye's wards in the Medical College Hospital, Calcutta, during the past ten years. Of these cases, five were operations for aneurysm and one for recurrent hæmorrhage. The wards contain eighty beds, and as but five cases of aneurysm entered them in ten years, the disease must be more rare in Lower Bengal than in most parts of Europe. In three of the five cases of aneurysm the treatment was commenced with com pression, but from various causes this had to be abandoned in favour of ligature. All these cases so treated recovered without any trouble. In a fourth ease the artery was ligatured without any previous compression. This case also ultimately recovered, but the leg was on the verge of gangrene. Mr. Raye's cases are too few to draw reliable conclusions from, but the inference seems to be that compression is still the safest mode of treatment, and that even when it has ultimately to be abandoned its temporary use is of advantage in so far as it prepares the way for establishment of the collateral circulation.

CASE 1.-A Hindoo male, aged thirty, of spare habit, was admitted with an aneurysm in the left axilla about the size of an orange. Duration four months. Pressure over the subclavian easily controlled the pulsation. Digital pressure was therefore attempted, and kept up for about two hours. $O$ wing to lack of proper assistants this treatment had to be abandoned. The aneurysm felt slightly firmer after the pressure. Two days later the subclavian was expose 1 and tied in its third stage. The vein gave no trouble. The artery was ligatured with catgut, tied firmly. Palsation at once ceased in the trmour, and did not return. A catgut drain was introduced into the wound, which was then closed. The wound healed by first intention. The operation was performed on the $22 \mathrm{nd}$ of Ostober, 1883 , and the patient discharged cured Feb. 1st, 1884

CASE 2.-A Earasian male, aged thirty-three, healthy, of slight build. Three months previous to admission he slipped in crossing a threshold, and felt immediate pain in the back of the knee. A few days later he noticed a swelling in the popliteal space, which slowly increased until the time of his coming to hospital, when it was found to be as big as a hen's egg. It was extremely painful and caused sleepless nights. Compression of the femoral stopped the pulsation, and likewise gave immediate relief from pain. Digital compression of the femoral was carried out by relays of students for three hours. The patient, a nervous, irritable man, then absolutely refused to allow this treatment to be continued, although his pain was thereby much relieved. Two days later he was persuaded to allow another trial of compression, but after a couple of hours it had to be discontinued on his refusal to submit further. After an interval of three days more a Carte's compressor was applied, but this the man at once rejected. Some benefit resulted from these compressions, as the tumour was rather more firm. Ultimately the superficial femoral was tied with catgut, the ligature being tightly drawn. A small catgut drain was placed in the wound, which was then closed. Pulsation in the tumour ceased at once, and did not recur. The dressings were removed on the eighth day, when the wound was found healed. The patient recovered without any troublesome symptom. He was seen two years later. A hardness about the size of an almond could be felt in the popliteal space. The leg was somewhat wasted, but in all respects serviceable. Operation performed Jan. 21st, 1885 ; discharged April 9 th, 1885.

CASE 3.-A Hindoo male, aged thirty.five, greatly weakened by chronic malaria, admitted with a strongly pulsating aneurysm occupying the outer two-thirds of the iliac region parallel with and limited by Poupart's ligament, and about the size of a small cocoanut. The upper part of the tumour was in contact with the lower border of the greatly enlarged spleen. The patient stated that two months previously to his admission he had to walk some fifty miles over rough roads, suffering great fatigue. Two days subsequently he noticed a small lump above Poupart's ligament. This gave rise to an acute "bursting" pain. For a month the swelling remained stationary, but during the three weeks preceding his coming to hospital it rapidly increased in size. It continued intensely painful throughout its progress. The man was kept at absolute rest for a 
week after admission, by which time his general condition had improved, and the pain bad slightly diminished. Treatment was then commenced under chloroform by Reid's method, an Esmarch's bandage being applied from the toes up to, but not over, the aneurysm, and a tourniquet placed on the aorta, controlling as far as possible the flow of blood in to the tumour. Within twenty minutes alarming syncope ensued, and the operation had to be abandoned. Next day the pain, hitherto excruciating, had almost ceased. The tumour was more firm, and somewhat shrunken possibly. No further improvement followed. Having waited for a fortnight's complete rest, the compression was again similarly applied, but this time it was found possible to arrange the abdominal tourniquet so as to press on the iliac artery only, and not on the aorta, the spleen having diminished sufficiently to admit of the pad being so adjusted. Complete compression was kept up for one hour and three quarters, when it was thought advisable to discontinue it. No benefit resulted from this second compres. sion. After a rest of another fortnight the com. pression was again applied as before, this time for an hour and $a$ half. No observable benefit followed. Three trials of compression having been made with but little benefit, the external iliac was ligatured with kangaroo tendon tightly tied. The enlarged spleen limited the field of operation but did not cause any serious difficulty. A rubber drainage-tube was inserted and the wound closed. Severe cramp in the limb followed and lasted several hours. Pulsation did not return at any time in the tumour. Dressings were changed on the seventh day, when the tube was removed. The wound had then united except where the drain lay. This patient was detained in hospital for several weeks on account of his malaria and splenic enlargement; the tumour had then contracted to the size of a hen's egg and felt solid. Operation performed Nov. 3rd, 1887 ; patient discharged cured on Dec. 31st, 1887.

CASE 4.-An able-bodied Mohammedan seaman, aged thirty-two, admitted with an oval-shaped aneurysm tensely filling the left popliteal space. The man was in a state of extreme nervous excitement, restless and tossing from side to side; complaining of general discomfort and of great pain in the leg. He was kept under the influence of opium for a day, and the superficial femoral was ligatured on the following morning with kangaroo tendon. The knot was drawn with (it was believed) sufficient force to close the vessel but not to rupture its coats. The wound was then closed. When the first dressings were removed on the eighth day union had taken place. The pain in the limb at once ceased, but the nervous excitement continued for several days. Gangrene threatened, and for the first week the nourishment of the limb was imperilled. The loss of vitality finally limited itself to four patches of the skin on the front of the leg and one on the dorsum of the foot, each about the size of a shilling. Later on the distal phalanx of the great toe was found to have necrosed. The healing of these dead patches delayed the patients' discharge for several weeks. Some shrinking of the foot and stiffness of its joints remained, and still continues (six months after the operation). Operation performed on Aug. 30th, 1890 ; patient discharged cured Nov. 25 th, 1890.

CAse 5.-A Hindoo male, aged fifty, admitted with an aneurysm of the left side of the neck. It filled up the hollow behind the lower jaw, and extended from the level of the hyoid bone to the mastoid process. On opening the mouth, it could be seen pulsating violently and bulging the wall of the pharynx inwards. It caused severe pain radiating up the side of the head, and a most distressing loud buzzing noise was also complained of. The common carotid was tied with kangaroo tendon, the ligature was fastened with slight tension with a view to closing, but not rupturing the coats of the artery. The jugular vein was not seen during the operation. The wound healed by first intention. The pain and the buzzing noise ceased at once. No cerebral symptom occurred. Operation performed on Aug. 30th, 1890.

CASE 6. -A Mohammedan female, aged fifty, admitted for recurrent hæmorrhage from a sloughing wound of the palm, inflicted eight days before she came to hospital. The state of the wound precluded all possibility of securing the vessel in the palm. The brachial was therefore ligatured. A high bifurcation was met with, and both trunks were tied with catgut. The wound healed by first intention. Operation performed on Feb. 24th, 1886; patient discharged cured on March 14th, 1886.
Remarks by Mr. O'ConNel, RAYE.-In two of these cases an attempt was made to tie the ligature with force only sufficient to close the lumen of the artery and not to rupture its coats. I cannot say whether this was actually done or not, for I do not possess tactus eruditus of sufficient refinement to allow me to speak with confidence. My im pression is that it must be an extremely difficult thing for any operator to tell by the touch what amount of damage his ligature has inflicted on the vessel.

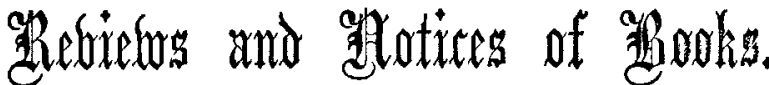

Carlsbad; its Thermal Springs and how to use them. By J. KRAUS, M.D. Fourth Edition, Revised and Enlarged. London: Kegan Paul, Trench, Trübner, and Co. 1891.

DR. Kraus issues the fourth edition of his well-known work on Carlsbad, with a prefatory note by Sir Joseph Fayrer, in which that distinguished physician says that "he holds Carlsbad, with its discipline and its waters, to be a remedy of great value, not only in many of the functional disorders of this country, but especially in a large number of those which obtain among persons who have resided in India or the tropics, and have suffered from the effects of their climates." Of the popularity of the famous Bohemian spa it is needless to speak. Carlsbad is probably the most popular watering-place on the Continent, and if our ows countrymen have shown less appreciation of it than Germans, Austrians, Poles, Russians, and Jews, yet the number of English resorting thither is already large, anc tending constantly to increase. Fashion counts for so much in the selection of a spa, and is so often capricious in its favours, that it is satisfactory to note the strong position occupied by a resort possessing such genuine merits, and at which so much sound therapeutical work is done, as Carlsbad. We may pass over the historical and antiquarian particulars in the work before us-details which often figure somewhat too largely in books upon health resorts, -and pass at once to the important question of the mineral constituents of the Carlsbad waters. In generat terms it may be said that the numerous Carlsbad springs possess practically the same characteristics. They are all hot, the temperature varying in the different springs from about $120^{\circ}$ to $160^{\circ} \mathrm{F}$., the extremes being $95^{\circ}$ and $162^{\circ}$. They are all strongly mineralised, containing about forty-one or forty-two grains of solid constituents per pound of water, and the chief ingredients are sulphate of soda, carbonate of soda, chloride of sodium, carbonate of lime, and sulphate of potash. The chief point of difference in the various springs is in the amount of free carbonic acid present, the cooler springs possessing more, and the hotter springs less, of this gas. In addition to free carbonic acid gas, the springs contain a large quantity of this gas in varying degrees of combination.

In addition to the drinking waters, Carlsbad has ample facilities for bathing. There are mineral baths at a tem. perature of $90^{\circ}$ to $100^{\circ} \mathrm{F}$., peat baths, the materials for which are brought by rail from Franzensbad, vapour and douche baths, steel baths, and acidulous baths. Carlsbad is also fortunate in possessing in the Giessh übler-Sauerbrunn an unlimited supply of an agreeable effervescing alkaline water suitable for table use. It contains a very large quantity of carbonic acid gas, both free and in combination.

The Carlsbad waters are especially recommended in. abdominal complaints in general, except acute fevers, malignant degenerations, and tumours. They are also. valuable in chlorosis, adiposis, gout, malaria, and diabetes, when not too far advanced. Dr. Kraus has also found great advantage from the waters in cases of chronic gastric 